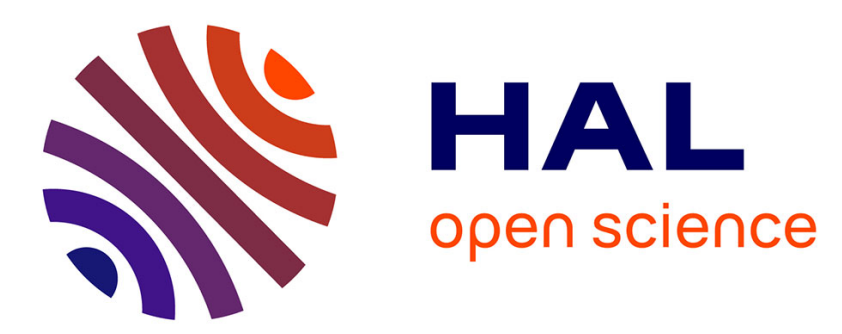

\title{
Ultrasound-induced coalescence of free gas microbubbles
} Michiel Postema, Philippe Marmottant, Charles T Lancée, Michel Versluis, Sascha Hilgenfeldt, Nico de Jong

\section{To cite this version:}

Michiel Postema, Philippe Marmottant, Charles T Lancée, Michel Versluis, Sascha Hilgenfeldt, et al.. Ultrasound-induced coalescence of free gas microbubbles. IEEE Ultrasonics Symposium, 2004, Apr 2004, Montréal, Canada. pp.1-4, 10.1109/ULTSYM.2004.1417653 . hal-03193406v2

\section{HAL Id: hal-03193406 https://hal.science/hal-03193406v2}

Submitted on 11 Apr 2021

HAL is a multi-disciplinary open access archive for the deposit and dissemination of scientific research documents, whether they are published or not. The documents may come from teaching and research institutions in France or abroad, or from public or private research centers.
L'archive ouverte pluridisciplinaire HAL, est destinée au dépôt et à la diffusion de documents scientifiques de niveau recherche, publiés ou non, émanant des établissements d'enseignement et de recherche français ou étrangers, des laboratoires publics ou privés. 


\title{
Ultrasound-induced Coalescence of Free Gas Microbubbles
}

\author{
Michiel Postema* ${ }^{* \dagger}$, Philippe Marmottant ${ }^{\ddagger}$, Charles T. Lancée*, Michel Versluis ${ }^{\ddagger}$, \\ Sascha Hilgenfeldt ${ }^{\ddagger}$ and Nico de Jong ${ }^{* \dagger \ddagger}$ \\ *Dept. of Experimental Echocardiography, Thoraxcentre, Erasmus MC, Rotterdam, The Netherlands \\ ${ }^{\dagger}$ Interuniversity Cardiology Institute of the Netherlands (ICIN), Utrecht, The Netherlands \\ ${ }^{\ddagger}$ Physics of Fluids group, Faculty of Science and Technology, University of Twente,
} Enschede, The Netherlands

\begin{abstract}
When gas bubbles collide, the following stages of bubble coalescence have been reported: flattening of the opposing bubble surfaces prior to contact, drainage of the interposed liquid film toward a critical minimal thickness, rupture of the liquid film, and formation of a single bubble. During insonification, expanding contrast agent microbubbles may collide with each other, resulting in coalescence or bounce.

In this study, we investigate the validity of the film drainage formalism for expanding free bubbles, by subjecting rigid-shelled contrast agent microbubbles to ultrasound, in order to release gas, and photograph the coalescence of these free gas bubbles. As with colliding bubbles, bubble surface flattening is related to the Weber number. Only inertial film drainage between free interfaces explains the observed coalescence times. In accordance with theory, smaller bubble fragments coalesce on very small time scales, while larger bubbles bounce off each other.
\end{abstract}

\section{INTRODUCTION}

When two gas bubbles collide or are driven into each other, coalescence into a single bubble may result. The following stages of bubble coalescence have been identified ( $c f$. Figure 1): flattening of the opposing bubble surfaces prior to contact, drainage of the interposed liquid film toward a critical minimal thickness, rupture of the liquid film, and formation of a single bubble. If the critical thickness is not reached during collision, the bubbles bounce off each other instead.

Free gas bubble coalescence after collision has been studied extensively [1], [2], [3], [4], [5], [6]. With ultrasound contrast agents, microbubble coalescence has been observed during ultrasound insonification, when expanding microbubbles come into contact with each other [7], [8], [9]. With the aid of highspeed photography, we previously investigated the coalescence times of insonified soft-shelled microbubbles. We compared observed coalescence times to calculated film drainage times, based on the Reynolds equation [10] for no-slip interfaces and on the drainage equation for free interfaces. We concluded that the bubbles behaved as if they had free interfaces like free gas bubbles [8]. However, to support this conclusion, we should investigate the validity of the drainage equations for expanding bubbles. In this study we do so, by subjecting rigid-shelled contrast agent microbubbles to ultrasound, in order to release gas, and investigate the coalescence of these free gas bubbles. We compare these times to the computations.

Previously, we reported on ultrasound-induced gas release from contrast agent microbubbles [7]. This so-called sonic
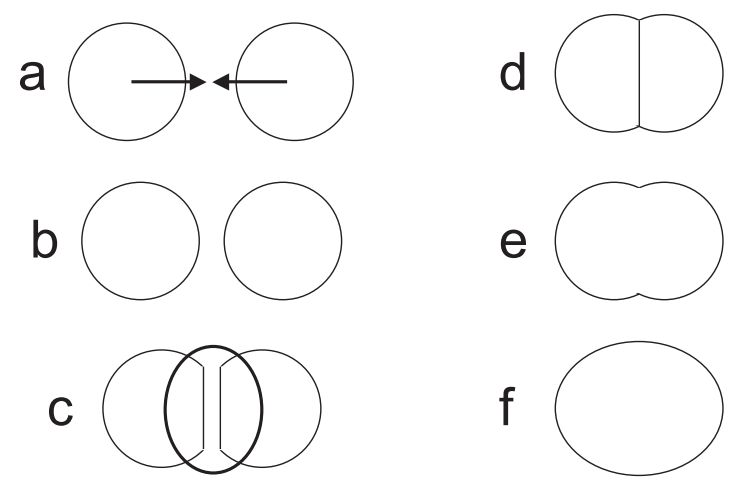

Fig. 1. Schematic representation of colliding bubbles or droplets: approach (a-b), flattening of the interposed film (c), drainage to a critical thickness (d), film rupture (e), and formation of a single bubble (f).

cracking has been observed with microbubbles with rigid encapsulations.

\section{THEORY}

Flattening of the opposing bubble surfaces occurs in case of colliding bubbles with constant volumes, if the bubble system has a Weber number We $\gtrsim 0.5$ [5]. If We $\lesssim 0.5$, the bubbles will coalesce without the prior formation of an interposed film. The Weber number for a fluid containing two bubbles with radii $R_{1}$ and $R_{2}$, respectively, is given by the inertial force relative to the surface tension force:

$$
\mathrm{We}=\rho u^{2} / \frac{\sigma}{R_{\mathrm{m}}},
$$

where $u$ is the relative approach velocity, $\rho$ is the fluid density, $\sigma$ is the surface tension, and $R_{\mathrm{m}}$ is the mean bubble radius for which holds:

$$
\frac{2}{R_{\mathrm{m}}}=\frac{1}{R_{1}}+\frac{1}{R_{2}} .
$$

We propose to extend this criterium to approaching walls of expanding bubbles. Because the radius and with it the approach velocity of oscillating bubbles change during a cycle, so does the Weber number. The approach velocity for expanding bubbles with a constant center-to-center distance is

$$
u=\dot{R}_{1}+\dot{R}_{2} \text {. }
$$


If the Weber number is low, bubble coalescence will always occur, without flattening of the adjacent surfaces prior to contact [5]. In the high Weber number regimen, coalescence is determined by a second step, after flattening: film drainage. When the expansion time is less than the time needed for film drainage, the bubbles bounce off [11].

The radial velocity of the liquid in the film is a combination of a plug flow driven by the motion of the interfaces, and a laminar velocity profile (analogous to Poiseuille flow) driven by the radial pressure gradient [12], [13]. If the bubble surfaces consist of a high concentration of surfactant, on our working scales the interfaces are to be considered immobile (no-slip) [14]. In the case of no-slip interfaces, the interfacial tangential velocity is zero, so the plug flow contribution is zero [12]. In the case of free interfaces, the Poiseuille contribution to the drainage flow becomes negligible [14], [12]. The film drainage time for free radial surfaces is approximated by the equation [15], [16]:

$$
\tau_{\mathrm{d}} \approx R_{\mathrm{f}} \sqrt{\frac{\rho}{8 p}} \log \left(\frac{h_{\mathrm{i}}}{h_{\mathrm{c}}}\right),
$$

where $\eta$ is the viscosity of the fluid, $\rho$ is the density of the fluid, $R_{\mathrm{f}}$ is the film radius, $h_{\mathrm{i}}$ is the initial thickness, $h_{\mathrm{c}}$ is the critical film thickness, at which the film ruptures, $p$ and is the pressure difference between film and surrounding fluid which is taken

$$
p=\sigma\left(\frac{1}{R_{1}}+\frac{1}{R_{2}}\right) .
$$

In our computations, we take

$$
\begin{aligned}
\eta & =0.001 \mathrm{~Pa} \mathrm{~s}^{-3}, \\
\rho & =998 \mathrm{~kg} \mathrm{~m}^{-3}, \\
\sigma & =0.072 \mathrm{~N} \mathrm{~m}^{-1}, \text { and } \\
h_{\mathrm{c}} & =10 \mathrm{~nm} .
\end{aligned}
$$

Flattening takes place when:

$$
\dot{R}_{1}+\dot{R}_{2} \gg \frac{\mathrm{d} h}{\mathrm{~d} t},
$$

whereas the flat film drainage happens in the next stage, when

$$
\dot{R}_{1} \approx \dot{R}_{2} \approx 0 .
$$

Thus, during drainage, on our timescales, we may take $p$ and $R_{\mathrm{f}}$ constant over time [8].

Repeated coalescence and fragmentation has been reported [8]. Fragmentation is the fission of a bubble into smaller bubbles. It occurs around peak contraction, when the bubble collapse is driven by inertial forces, since the inward acceleration continues to increase as the bubble approaches its minimum radius and suddenly changes sign as the bubble begins a rebound [17]. The number of fragments into which a bubble breaks up has been related to the dominant spherical harmonic oscillation mode $n$ [18], [9]. The estimated number of fragments $N$ is

$$
N \approx n^{3} .
$$

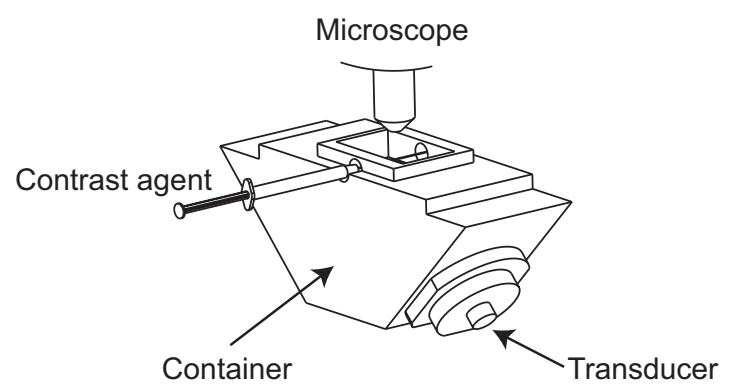

Fig. 2. Overview of the experimental bubble-system.

\section{EXPERIMENTAL METHODS}

Our experimental setups for taking pictures of insonified contrast bubbles were as described in [19] and in [20]. An overview of the bubble-system is shown in Fig. 2. A $\oslash 200 \mu \mathrm{m}$ capillary tube, through which contrast agent was flowing, was fixed in the focal area of a v389-su $500 \mathrm{kHz}$ or a v397SU $2.25 \mathrm{MHz}$ single-element transducer (Panametrics Inc., Waltham, MA). The optical observations of the insonified ultrasound contrast agent were recorded with the Brandaris128 system, operating at speeds up to $15 \mathrm{MHz}$.

The free gas bubbles were released from PB127 (POINT Biomedical Corporation, San Carlos, CA). PB127 consists of bilayered microspheres encapsulating nitrogen bubbles with a mean diameter of $4 \mu \mathrm{m}$. The outermost layer is albumin and the inner layer is composed of a biodegradable polymer. The content of a PB127 vial was resuspended in $5 \mathrm{ml}$ of deionized water, and shaken gently for 20 seconds before further dilution. Diluted PB127 was inserted through the capillary tube using a syringe pressed by hand. It was insonified by 6 or 8 cycles of $500 \mathrm{kHz}$ (period $T_{\mathrm{p}}=2 \mu \mathrm{s}$ ), or by 8 cycles of $1.7 \mathrm{MHz}$ $\left(T_{\mathrm{p}}=0.6 \mu \mathrm{s}\right)$ ultrasound at acoustic pressures corresponding to mechanical indices between 0.3 and 1.9 .

\section{RESULTS AND DISCUSSION}

The same stages of coalescence described for colliding bubbles could be distinctly observed with expanding free gas microbubbles and soft-shelled microbubbles. Examples of softshelled microbubble coalescence were shown in [7], [8], [21].

As with encapsulated bubbles, coalescence and bounce were regularly observed. For the interacting free gas microbubbles with flattened interfaces observed in the optical images, We 1 . We obtained 9 image sequences where free bubble coalescence times and the bubble dimensions could be measured. The times between flattening of the free bubble surfaces and coalescence are less than $0.3 \mu \mathrm{s}$, for expanded gas bubbles with diameters between 3 and $5 \mu \mathrm{m}$.

Figure 3 shows an example of gas release from a PB127 bubble. Each frame corresponds to a $23 \times 23 \mu \mathrm{m}^{2}$ area. Four PB127 bubbles are in the field of view. After ultrasound arrival (frame 13) the bubbles contract. During expansion, gas escapes from the $\oslash 3 \mu \mathrm{m}$ upper bubble (16-19). The free gas is seen detached in frame 21. It expands (22-25) and starts to contract (26). Close to its minimum, the free gas bubble 
splits up into multiple fragments (27). During expansion, the fragments appear to have coalesced into two separate bubbles (28-29). These bubbles collide during expansion (30). The Weber number, calculated from the bubble wall velocity (2930) $u \simeq \frac{2 \mu \mathrm{m}}{0.1 \mu \mathrm{s}}$, is $\mathrm{We}>20 \gg 0.5$, which agrees with the flattening of the bubble surfaces. Computing equation (4) with $R_{1}=3.9 \mu \mathrm{m}, R_{2}=3.4 \mu \mathrm{m}$, and $R_{\mathrm{f}}=2.1 \mu \mathrm{m}$ yields

$$
\tau_{\mathrm{d}}=0.6 \mu \mathrm{s} \approx T_{\mathrm{p}} .
$$

This long drainage time accounts for the observed bounce (3032). Close to maximal contraction (33), the two bubbles fragment. During expansion, the fragments are seen to coalesce (35-37) into two bubbles of perpendicular orientation. Again, these bubbles bounce (38-39), fragment (40), and coalesce into two bubbles perpendicular to their mother bubbles (41). This process repeats itself, until insonification ends (66). The two remaining free gas bubbles still pulsate, and collide while doing so. These collisions may possibly be attributed to secondary radiation forces. In frame 82 , the bubbles coalesce. This coalescence is induced by the pressure field exerted by the bubbles themselves. The remaining bubble has a maximum of $3.8 \mu \mathrm{m}$. It pulsates at $1.7 \mathrm{MHz}$. Hence, the pulsations may be attributed to reflections.

Note that the radii of the bubble fragments after a fission event are all: $R_{i}<1 \mu \mathrm{m}$. From equations (4)-(6), using the empirical relation [8]:

$$
R_{\mathrm{f}} \approx \frac{2}{3} R_{i}
$$

it follows that the drainage times, and with it the fragment coalescence times are:

$$
\tau_{\mathrm{d}}<0.1 \mu s,
$$

consistent with the observed fast coalescence of fragments in the space of an inter-frame distance $(0.1 \mu \mathrm{s})$.

The bouncing bubbles in Fig. 3 might be considered as an $n=2$ spherical harmonic mode system. This is supported by the alternating perpendicular orientations observed. The estimated number of fragments $\left(N \approx 2^{3}=8\right)$ cannot be verified by these observations, because right after fragmentation, the fragments are too small to be observed.

\section{CONCLUSIONS}

Expanding gas bubble coalescence has been observed with released gas. Bubble surface flattening is observed to be consistent with the Weber number criterium for colliding bubbles.

Calculated drainage times from an inertial drainage model assuming clean, stress-free interfaces are consistent with the observations: For large (fully expanded) bubbles the drainage times are too large (larger than a bubble oscillation cycle) to allow for film drainage and coalescence. Smaller microbubble fragments, however, easily coalesce on very short timescales.

\section{ACKNOWLEDGMENTS}

This work has been supported by the Technology Foundation STW (RKG.5104). We are indebted to Andrea Prosperetti for very helpful discussions and thank POINT Biomedical Corporation for supplying PB127.

\section{REFERENCES}

[1] G. Marrucci, "A theory of coalescence," Chem. Eng. Sci., vol. 24, pp. 975-985, 1969.

[2] D. S. Dimitrov and I. B. Ivanov, "Hydrodynamics of thin liquid films. On the rate of thinning of microscopic films with deformable interfaces," J. Colloid. Interf. Sci., vol. 64, no. 1, pp. 97-106, 1978.

[3] I. B. Ivanov, D. S. Dimitrov, and B. P. Radoyev, "Generalized equations of thin films hydrodynamics and their application to the calculations of the thinning rate of films with non-deformed surfaces," Kolloid. Zh., vol. 41, no. 1, pp. 36-42, 1979.

[4] C. Y. Lin and J. C. Slattery, "Thinning of a liquid film as a small drop or bubble approaches a fluid-fluid interface," AIChE J., vol. 28, no. 5, pp. 786-792, 1982.

[5] A. K. Chesters and G. Hofman, "Bubble coalescence in pure liquids," Appl. Sci. Res., vol. 38, pp. 353-361, 1982.

[6] P. C. Duineveld, Bouncing and coalescence of two bubbles in water. Enschede: PC Duineveld, 1994.

[7] M. Postema, A. Bouakaz, C. T. Chin, and N. de Jong, "Optically observed microbubble coalescence and collapse," Proc. IEEE Ultrason. Symp., pp. 1900-1903, 2002.

[8] M. Postema, P. Marmottant, C. T. Lancée, S. Hilgenfeldt, and N. de Jong, "Ultrasound-induced microbubble coalescence," Ultrasound Med. Biol., submitted, 2004.

[9] M. Postema, Medical Bubbles. Bergschenhoek: Michiel Postema, 2004.

[10] O. Reynolds, "On the theory of lubrication and its application to Mr. Beauchamp Tower's experiments, including an experimental determination of the viscosity of olive oil," Philos. Trans. Roy. Soc. A, vol. 177, pp. 157-234, 1886.

[11] R. V. Chaudhari and H. Hofmann, "Coalescence of gas bubbles in liquids," Rev. Chem. Eng., vol. 10, no. 2, pp. 131-190, 1994.

[12] E. Klaseboer, J. P. Chevaillier, C. Gourdon, and O. Masbernat, "Film drainage between colliding drops at constant approach velocity: experiments and modeling," J. Colloid. Interf. Sci., vol. 229, pp. 274-285, 2000.

[13] D. F. Young, B. R. Munson, and T. H. Okiishi, A Brief Introduction to Fluid Mechanics, 2nd ed. New York: John Wiley \& Sons, 2000.

[14] L. van Wijngaarden, "The mean rise velocity of pairwise-interacting bubbles in liquid," J. Fluid Mech., vol. 251, pp. 55-78, 1993.

[15] R. D. Kirkpatrick and M. J. Lockett, "The influence of approach velocity on bubble coalescence," Chem. Eng. Sci., vol. 29, pp. 2363-2373, 1974.

[16] L. Hagesæther, Coalescence and break-up of drops and bubbles. Trondheim: Norwegian University of Science and Technology, 2002.

[17] J. E. Chomas, P. Dayton, D. May, and K. Ferrara, "Threshold of fragmentation for ultrasonic contrast," J. Biomed. Opt., vol. 6, no. 2, pp. 141-150, 2001.

[18] C. E. Brennen, "Fission of collapsing cavitation bubbles," J. Fluid Mech., vol. 472, pp. 153-166, 2002.

[19] C. T. Chin, C. Lancée, J. Borsboom, F. Mastik, M. Frijlink, N. de Jong, M. Versluis, and D. Lohse, "Brandaris 128: a 25 million frames per second digital camera with 128 highly sensitive frames," Rev. Sci. Instru., vol. 74, no. 12, pp. 5026-5034, 2003.

[20] M. Postema, A. van Wamel, C. T. Lancée, and N. de Jong, "Ultrasoundinduced encapsulated microbubble phenomena," Ultrasound Med. Biol., vol. 30, no. 6, pp. 827-840, 2004.

[21] M. Postema, A. Bouakaz, C. T. Chin, and N. de Jong, "Simulations and measurements of optical images of insonified ultrasound contrast microbubbles," IEEE Trans. Ultrason., Ferroelect., Freq. Contr., vol. 50, no. 5, pp. 523-536, 2003.

Michiel Postema e-mail: m.postema@erasmusmc.nl 


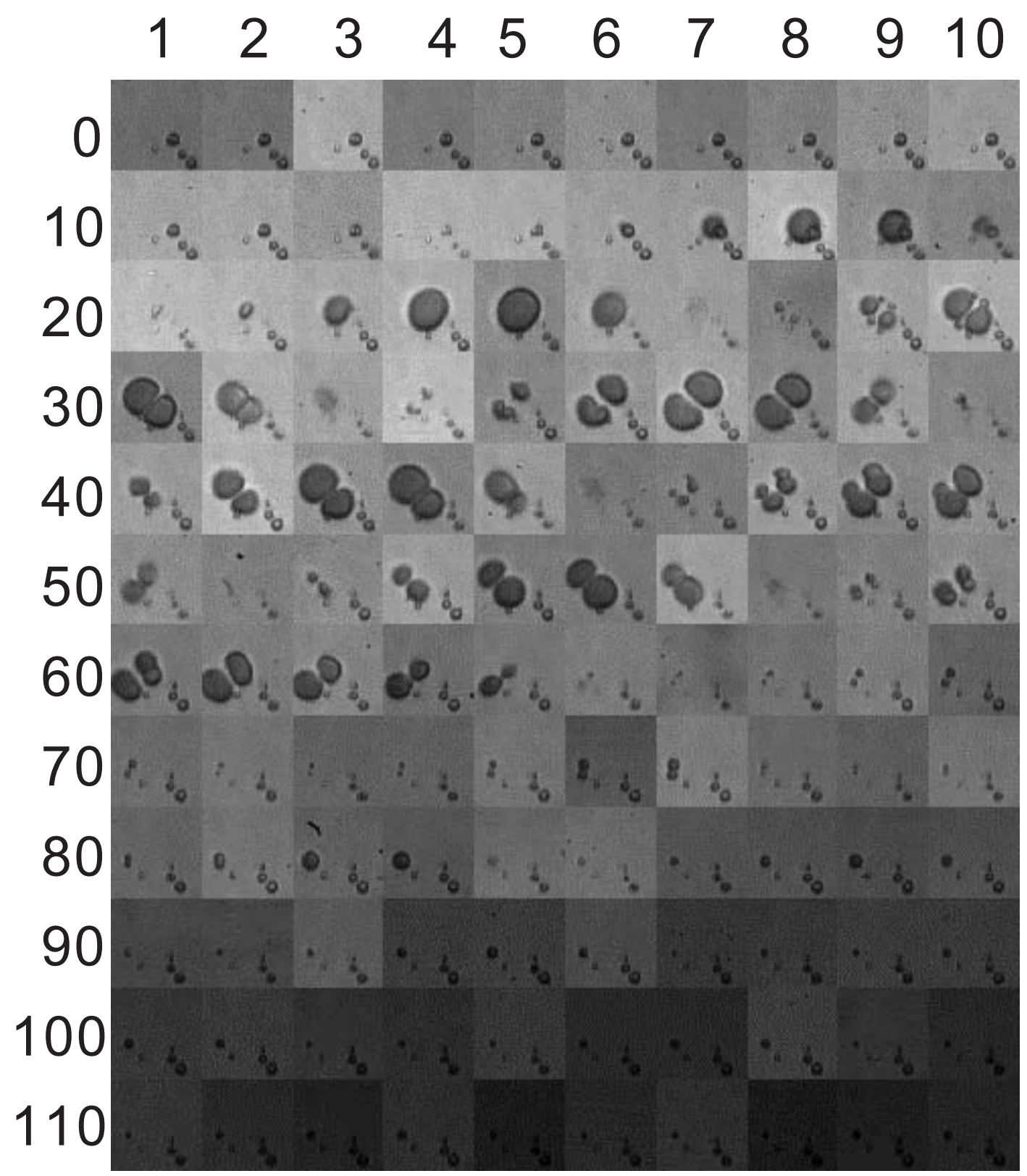

Fig. 3. Gas release at $1.7 \mathrm{MHz}$ from $\mathrm{PB} 127$ microbubbles, captured at 10 Mfps. Each frame corresponds to a $23 \times 23 \mu \mathrm{m}^{2}$ area. Spontaneous coalescence of free gas microbubbles takes place in frames 81-83. 